\title{
Functionality Advancement of Poorly Soluble Drug by Comparative Study of Solubilizing Techniques with Molecular Simulation to in vivo evaluation
}

\author{
Poonam Mogal
}

\author{
Department of Pharmaceutics, N.D.M.V.P.'s College of Pharmacy, Gangapur Road, \\ Nashik, 422002. Maharashtra, India.
}

\begin{abstract}
This study aimed at the functionality advancement of poorly soluble model drug i.e. Cefixime with HPBCD using comparative study of different solubility enhancement methods. CFX formulations with hydroxypropyl- $\beta$-cyclodextrin (HP- $\beta-C D$ ) prepared by different solubility enhancement methods viz.,physical mixture, kneading method, solvent evaporation, spray drying, lyophilization/freeze drying, microwave irradiation method and compared their solubility enhancement as well as dissolution.

The optimized complexes characterized using ATR, 1H-NMR, ROSEY, XRD, SEM and DSC for confirming the complexation. In-vivo pharmacokinetic studies were performed using male wister rats $(\mathrm{N}=6)$ through oral route.

Hydroxy propyl Beta cyclodextrin inclusion complexation prepared by spray drying method was found to be optimized for complexation with cefixime.

Spray drying method was found to be better than other complexation techniques on basis of in-vitro dissolution studies. ATR, NMR, XRD, and DSC confirmed the formation of complex.

The in silico anticipations for mode of incorporation were in agreeing with the experimental proton NMR measure.

The bioavailability of cefixime was found to be enhanced more than 3 times. In comparison to pure drug, statistically significant increase in the oral bioavailability was obtained with the HP- $\beta$-CD inclusion complex which was prepared by spray drying technique, with $347 \%$ increase in terms of Cmax.

This comparative study gave idea about best suited method for solubility enhancement along with molecular docking and NMR studies for anticipating mode of inclusion \& thus, ultimately, improving the oral bioavailability of CFX.
\end{abstract}

Keywords : Cefixime, solubility, Inclusion complexation, HP- $\beta-C D$, cyclodextrin.

\section{Introduction}

One of the methods to enhance solubility of poorly water soluble is that the inclusion of the drug as guest into cyclodextrin cavity as host, that offers various further benefits, like improvement of the stability, safety, 
organoleptic properties, etc. of the developed formulation ${ }^{1}$. As a result of these benefits there are greater than forty pharmaceutical products comprising cyclodextrin are obtainable in the market, where the number of them use $\beta C D$ and its derivatives of high aqueous solubility like $\mathrm{HP} \beta C D, M \beta C D$, as well as $\mathrm{SBE} \beta C \mathrm{D}^{2}$. In addition, it is interesting to note that, Cagno has even reviewed cyclodextrin's "potential as active pharmaceutical ingredients (APIs) for the treatment of several illnesses." The quantity of cyclodextrins in a final drug formulation should be reduced because of its toxicity, cost, and dosage, and it is attained by the choice of a best suited cyclodextrin type, with optimized complexation technique to prepare formulation ${ }^{3}$. Therefore, this study was directed to illuminate solubility enhancement of the poorly soluble drug, Cefixime by complexing agent i.e. 2-hydroxy)propyl- $\beta$-cyclodextrin (HPBCD), having good aqueous solubility with low toxicity profile ${ }^{3}$ Cefixime-cyclodextrin complexes were prepared by spray drying method, and their properties were compared with those prepared by solvent evaporation, co-grinding, microwave irradiation, freeze drying and physical mixing. Cefixime is undergoing clinical trials for several diseases like gonorrhea, Diarrhea, Mucopurulent cervicitis (MPC), Pyelonephritis, Urinary Tract Infections, Typhoid Fever, Chronic Kidney Disease, Osteomyelitis ${ }^{4,5}$. World Health Organization's Listed it as Essential Medicines, "the most effective and safe medicines" required in a health system ${ }^{6}$. Cefixime, first oral extended-spectrum cephalosporin antibiotic is often used for the treatment of the ear, UTI, and upper respiratory tract. The bactericidal mechanism of Cefixime is attributable to the inhibition of cell wall synthesis. It binds to at least one of the penicillin binding proteins (PBPs) that inhibiting the last transpeptidation step of the peptidoglycan synthesis within the bacterial cell wall. Hence, it turns to inhibition of biosynthesis\& stopping cell wall assembly leading to bacterial cell death.It is having only $40-50 \%$ oral bioavailability and its absorption is decreased when given with food ${ }^{7}$.

For bactericidal Cefixime, range of action involves several gram-positive \& gram-negative aerobic bacteria but its inactive against most anaerobic bacteria. It is also inactive against chlamydia, fungi, as well as viruses. Cefixime is having good efficacy verses a systemic infection i.e. typhoid fever. It is broad spectrum cephalosporin, but is very efficatious verses serovar Typhi also ${ }^{\mathbf{8 , 9}}$. Cefixime is prepared by chemical synthesis method is having empirical formula of $\mathrm{C} 16 \mathrm{H} 15 \mathrm{~N} 5 \mathrm{O} 7 \mathrm{~S} 2.3 \mathrm{H} 2 \mathrm{O}$ with the relative molecular mass of 507.49. It is (6R, 7R)-7-[2-(2-amino-4- thiazolyl)glyoxylamido]-8-oxo-3-vinyl-5-thia-1-azabicyclo [4.2.0]oct-2-ene-2carboxylic acid,7-(Z)-[o-(carboxy methyl)-oxime] trihydrate ${ }^{\mathbf{1 0}}$. It is is freely soluble in methanol and poorly soluble in water and has been reported as BCS class $2 / 4$ drug, with an oral bioavailability of $40-50 \%{ }^{11}$. Cefixime is available only in oral form as suspension and tablets ${ }^{\mathbf{1 0}}$. Cefixime, a third generation oral cephalosporin antibiotic, has poor lipophilicity as well as inadequate ionization at physiological $\mathrm{pH}$, when orally administered it is significantly absorbed unchanged ${ }^{\mathbf{1 2}}$. Bioavailability studies in man studied by Faulkner and in vitro experiments by Tsuji have shown that cefixime absorption is saturable ${ }^{13-15}$. Transport studies made by Dantzig, Kimura and Tsuji lead to the conclusion that common transport systems may be existed for cephalosporin antibiotics due to their similar chemical structure to that of dipeptides. More while, other studies by Dantzig suggested that simple diffusion into enterocytes and other researcher Inui suggested that paracellular pathway may also be involved for cefixime 6-19. $^{16}$.

Numerous formulation approaches have been investigated to overcome delivery barriers of Cefixime. Solid dispersion with Croscarmellose sodium ${ }^{20}$, Urea ${ }^{21}$, Gaur gum ${ }^{22}$, PVP K-30 ${ }^{23}$ are reported. Inclusion complexation with $\beta$-cyclodextrin ( $\beta-C D)$ and hydroxypropyl $\beta$-cyclodextrin (HPß-CD) have also been studied using solvent evaporation method ${ }^{24}$. The kinetic measurements of hydrolytic degradation of cefixime in Captisol complexation ${ }^{25}$ were explored. Nanoparticles using compritrol ATO 888 as the lipid core was formulated ${ }^{26}$. Other drug delivery systems like Nano carrier, Nanoparticles were also prepared using Fenugreek seed mucilage and Chitosan ${ }^{27}$. Dry emulsion of Cefixime was also prepared ${ }^{28}$. Effect of hydrophilic carrier on complexation of Cefixime has also been explored ${ }^{29}$. Ternary inclusion compound was made with L-arginine along with molecular modeling study using BCD ${ }^{30}$. Hence, for HPBCD complexation this study is not done yet. Literature survey states that very few experiments are carried out for meaningful comparison of solubility enhancement methods for Cefixime also its only restricted to in vitro studies. Studies done earlier for Cefixime judgment of solubility and solubility enhancement methods employed for that differ significantly in terms of polymer made use of and method utilized for solubility enhancement. Moreover, many of the reported studies were confined to in vitro assessment of formulations. This forbid substantive comparison of the versatile formulation approaches as well as methods employed for solubility enhancement in terms of their bioavailability benefit. Thus, the information associated with solubility improvement strategies differ considerably in polymer and methodology used and so we are unaware of the best. Hence, it's important to compare these various solubility improvement methods and to select best suited method for the formulation 
development of poorly soluble drugs like Cefixime. In the present study, we have employed different solubility enhancement methods viz., physical mixture, kneading method, solvent evaporation, spray drying, lyophilization/freeze drying, microwave irradiation method and compared their solubility enhancement as well as dissolution. Further, the oral bioavailability of best formulation was checked in male wister rats. The possible mechanism of inclusion of Cefixime into HPBCD cavity was also elucidated by molecular docking (in silico method) and $1 \mathrm{H}$ NMR, 2D ROSEY (experimental results). It would let a straightforward comparability of varied solubility enhancement techniques as well as improvement in oral bioavailability of the best formulation as compared to pure drug.

\section{Materials and methods}

\section{Materials}

Cefixime (CFX) andHP $\beta C D$ were kindly supplied by Glenmark pharmaceuticals, Sinnar, Nashik. All the reagents and solvents used or the experiments were of analytical grade.

\section{Methods}

\section{Phase solubility study}

In accordance with the method of Higuchi and Connors ${ }^{31}$, phase solubility studied was carried out. To $20 \mathrm{ml}$ aqueous solutions, conc. (1-0.01M) of HPBCD, drug was added in excess quantity. Then, it was stirred on magnetic stirrer and after that supernatant was taken and diluted appropriately after filtration and concentration of drug was checked by UV at $288 \mathrm{~nm}$.

\section{Preparation of Inclusion complexations}

\section{Physical mixtures}

Physical mixtures, in different ratios $(1: 1,1: 2)$ were prepared by mixing Cefixime with HPBCD for four-five min in a mortar till a uniform mixture was acquired. The mixtures obtained were passed through sieve (100 $\mu \mathrm{m}$ mesh) after which they were stored in a desiccator at normal temperature for further usage.

\section{Kneading method}

In this most common and simple method, Inclusion complexations with various ratios like $(1: 1,1: 2)$ were prepared. Firstly, HPBCD was added up in the mortar, then little quantity of methanol was add together during trituration for acquiring similar uniformity like slurry. And then gradually drug was added in the slurry with triturating it for one hour. Air drying was carried out for it at $25^{\circ} \mathrm{C}$ for $24 \mathrm{hr}$, powdered by sieving through sieve (No. 100) and after that it was kept in desiccator all over fused calcium chloride.

\section{Solvent evaporation method}

Tachibana and Nakamura described the solvent evaporation method for poorly soluble drug. Thus according to that Inclusion complexations in various ratios like $(1: 1,1: 2)$ were made by dissolving Cefixime (CFX) in Nonaqueous solvent whereas, HPBCD was made soluble in aqueous solvent. Dissolved drug solution was add together to hydrophilic water solution by constant stirring and add-on rate of 7-9 $\mathrm{ml} / \mathrm{min}$. Nonaqueous phase was evaporated in oven at $45-50^{\circ} \mathrm{C}$. The product was sieved through sieve (No. 100) \& further, it was kept in desiccator at room temperature till further analysis by UV.

\section{Lyophilization/Freeze-Drying Technique}

The Cefixime (CFX) and $\operatorname{HPBCD}(1: 1,1: 2)$ were accurately weighed when Cefixime (CFX) was made soluble in methanol \& polymer in aqueous solution. Methanolic solution was add up to water solution by uninterrupted stirring at the rate of $5-6 \mathrm{ml} / \mathrm{min}$. The nonaqueous solution (methanol) was removed by evaporation at room temperature. It was later lyophilised in Labconco lyophilizer (USA) after initial freezing with vacuum $0.040 \mathrm{mbar}$ (constant for all). The lyophilised product thus obtained was stored in desiccator at room temperature. 


\section{Microwave Irradiation Method}

The Cefixime (CFX) and $\operatorname{HPBCD}(1: 1,1: 2$, and 1:3) were accurately weighed. A homogeneous paste was made by adding Cefixime (CFX) \& HPBCD with minimal quantity of solvents (Ethyl alcohol: H2O, 1:1 $\mathrm{v} / \mathrm{v}$ ) inside vessel. The paste developed was subjected to microwave irradiation for 7-8 min at power of 510 watt in a microwave (oven) synthesizer (CATA-R, catalyst systems, Pune, India). Just a single beaker was set at once inside the microwave synthesizer. The specimens were presented to microwave radiation for 7-8 min. at that point, the beakers containing tests were kept up at normal temperatures to cool down. The ICs were gathered and put in desiccator for 24 hours and afterward the item was powdered utilizing mortar and pestle. The powdered product was sieved through sifter no. 100 and put away in desiccators over combined calcium chloride

\section{Spray drying}

Each solution for spray drying was prepared by adding different ratio of Cefixime and HPBCD (1:1, $1: 2)$ to $200 \mathrm{ml}$ of methanol. It was exposed to ultrasonication by a bath sonicator for about $10 \mathrm{~min}$. After that spray drying was carried out in a lab spray dryer model LU-222 Advanced (Labultima, Mumbai, India) with the drying capacity of $1 \mathrm{~L} / \mathrm{h}$. Parameters set for spray drying were like using $4 \mathrm{ml} / \mathrm{min}$ flow rate, $80^{\circ} \mathrm{C}$ of inlet temperature, with $60-70^{\circ} \mathrm{C}$ of outlet temperature, and aspirator value of about $60 \mathrm{~m}^{3} / \mathrm{h}$.

\section{Attenuated Total Reflectance Spectroscopy (ATR) analysis}

Supporting evidence for formation of inclusion complex can be acquired by IR spectroscopy. Attenuated total reflectance spectra of the drug samples were obtained on a Bruker Eco-ATR machine i.e. Attenuated total reflectance spectroscopy. For getting IR spectra, scanning was done over the wave number from 3600 to $400 \mathrm{~cm}^{-1}$.

\section{Differential Scanning Calorimetry (DSC)}

The possibility of any interaction between Cefixime \& HPBCD amid arrangement of IC was evaluated via DSC examination \& comparing it with that of pure Cefixime using DSC. DSC assessment was carried by differential scanning calorimeter (Lab Mettler Star SW 10). Heating of tests was done in an unsealed aluminium pan with heating a rate of $10^{\circ} \mathrm{C} / \mathrm{min}$ over a temperature from 25 to $300^{\circ} \mathrm{C}$ using a nitrogen rate of flow at 50 $\mathrm{ml} / \mathrm{min}$.

\section{X-ray Diffractometry (XRD)}

XRD spectrum of pure Cefixime and IC were taken at normal temperature with the help of Bruker's AXS D8-advance X-ray diffractometer. Irradiation of tests was carried out with mono-chromatized Cu KA2 emission at wavelength $1.5406 \mathrm{~A}^{0}$ and tests were mounted on zero-foundation test holder and subjected to a ceaseless scanning over angles of $3^{\circ}$ to $80^{\circ} 2 \theta$ at a stage size of $0.02^{\circ}$. The diffraction spectrums were gathered with voltage of $40 \mathrm{kV}$ and current of $35 \mathrm{~mA}$ individually. The examining rate was $2^{0}$ min- 1 .

\section{Scanning electron microscopy (SEM)}

The topography of pure Cefixime and spray dried IC was looked under a scanning electron microscope (SEM; JEOL model JSM -6390LV) operating at an excitation voltage of $15 \mathrm{kV}$.

\section{Saturated Solubility measurements of CFX}

As indicated by the strategy portrayed by Higuchi and J. Connors (1965), Solubility estimations were done. An excess quantity of inclusion complex was included $10 \mathrm{ml}$ distilled water, which was taken in test tubes. The tests were sonicated for $1 \mathrm{hr}$ at room temperature. From that point, the topped test tubes were shaken at 25 or $45 \pm 0.1^{\circ} \mathrm{C}$ for $24 \mathrm{hrs}$. In this manner, the suspensions were sifted through Whatmann filter paper no. 41 , and the separated solutions were analysed by UV-spectrophotometer at $288 \mathrm{~nm}$.

\section{Dissolution studies}

These studies were carried out by utilizing USP 8-station dissolution test assembly (Lab India) utilizing USP type II device. Dissolution study was done in a $900 \mathrm{ml}$ of $\mathrm{pH} 7.2$ buffer at $37 \pm 0.5^{\circ} \mathrm{C}$ at $100 \mathrm{rpm} .5 \mathrm{ml}$ tests were withdrawn at time interims of $5,10,15,20,30,45 \mathrm{~min}$. The volume of dissolution medium was conformed to $900 \mathrm{ml}$ to keep up sink conditions by supplanting every $5 \mathrm{ml}$ aliquot pulled back with $5 \mathrm{ml}$ of new pH 7.2 phosphate buffer. The drug's concentration in tests was dictated by measuring absorbance at $288 \mathrm{~nm}$. 
Aggregate percent drug release was ascertained at each time interim. Pure Cefixime was utilized as control for examination.

\section{Antimicrobial studies}

In vitro antibacterial investigations of ICs were done by disk diffusion technique verses Gram-positive species S. aureus and Gram-negative species E. coli and P. aerugenosa. The activities were compared against pure Cefixime (CFX). For this studies $\mathrm{MH}$ agar medium was used as nutrient medium. The petriplates were inoculated. Incubation of plates was done at $37^{\circ} \mathrm{C}$ for $24 \mathrm{hr} \&$ the zone of inhibition was measured in mm by zeta $\operatorname{sizer}^{32}$.

\section{Proton nuclear magnetic resonance (1H-NMR), and 2D NMR ROESY spectroscopy}

NMR experiments were performed in D2O with tetramethylsilane (TMS) as internal standard.1HNMR, 2D ROESY spectra in D2O of Cefixime and inclusion complexe were recorded with a Brucker AC $300^{\circ} \mathrm{C}$ NMR spectrometer apparatus operating at $300 \mathrm{MHz}$. For 2-dimensional (2D) ROESY experiment, samples were equilibrated for at least $24 \mathrm{hrs}$ at $300^{\circ} \mathrm{C}$.

\section{Molecular Docking Simulations ${ }^{34}$}

\section{Structure readiness}

The three-dimensional structure for BCD was downloaded from Protein Data Bank (PDB ID: 3CGT) and HPBCD was worked by including 2-hydroxypropyl group at suitable places of BCD (33). Every one of the structures was minimized energetically in Schrodinger Suite 2012 (Schrödinger, USA).

\section{Ligand arrangement}

The LigPrep procedure comprises of a progression of steps that perform changes, apply redresses to the structures, produce minor variant on the structures, dispense with undesirable structures, \& enhance the structures.

\section{Formation of host-guest complex}

Drug complexes with various HPBCD analogs were set up by manual method by setting the ligand in the hydrophobic cavity of the CDs in various orientations. As, the correct restricting mode was unknown different alignment of ligand in the cavity were attempted.

\section{Docking and Scoring Function}

This molecular docking instrument, Glide (Schrodinger Inc. U.S.A.) programming was utilized for ligand docking into the Protein binding pocket. Glide computations utilize an all-molecule force field for exact energy assessment. Docking simulations of compounds were carried out using the Glide (Schrodinger Inc. U.S.A.) software program that uses an 'Extra precision' Glide algorithm for docking ligand with the active site while the final scoring of docked ligand is carried out on the energy-minimized poses Glide Score scoring function.

\section{In-Vivo absorption study in wistar rats}

Wistar-strain rats of either sex (180-220 g) were made available by the Animal House, college of Pharmacy, Nashik, India. They were housed under normal laboratory conditions. Rats were maintained on standard rodent chow and tap water. The Institutional Animal Ethical Committee (IAEC/Feb.2016/08) gave approval for the experimental methods.

The rats were kept with empty food however, but they were given clear reach to water $24 \mathrm{~h}$ earlier the day of the experiment. 3 rats group were utilized in the study. Random assignment of 6 rats to each treatment group was done. To individual group oral administration of Cefixime aqueous suspension, Cefixime-ICs and third group was control group, was done. Sample of Cefixime powder $(8 \mathrm{mg})$, Cefixime-ICs (equivalent to Cefixime $8 \mathrm{mg}$ ), were mixed uniformly by accurately weighing \& dispersing it into water $(5 \mathrm{ml})$ for $30 \mathrm{~s}$ before 
to dosing. To rats, individual preparation was given utilizing oral gavage (an animal feeding needle). Blood samples $(0.5 \mathrm{ml})$ were collected via tail vein at 30,60,120, 240 and $360 \mathrm{~min}$ after oral administration into EDTA microcentrifuge tubes. Centrifugation of samples was done at $10,000 \mathrm{rpm} 10^{\circ} \mathrm{C}$ for $20 \mathrm{~min}$ where samples of plasma separated, and $0.5 \mathrm{ml}$ of cold acetonitrile add up to the plasma sample for precipitating the protein. The processed samples's centrifugation was again done at $10,000 \mathrm{rpm} 10^{\circ} \mathrm{C}$ for $20 \mathrm{~min}, \&$ supernatant liquid was stored in refrigerator until analyzed by HPLC ${ }^{35}$.

\section{HPLC analysis of plasma samples}

Standard solutions of $2,4,6,8$, and $10 \mu 1$ were prepared and calibration curve was plotted by HPLC. 20 $\mu \mathrm{l}$ of plasma sample was put in into HPLC. Concentration of Cefixime in plasma was then further determined by calculating area under curve of chromatogram.

HPLC (Binary HPLC pump provided with a UV visible 2000 detector, Waters 1525, Singapore) was utilized for the assessment of plasma samples. The sorting was performed using a Cosmosil C18 packed column $(250 \mathrm{~mm} \times 4.6 \mathrm{~mm}, 5 \mu \mathrm{m})$ at $276 \mathrm{~nm}$. The mobile phase consisted of a mixing of phosphate buffer having $\mathrm{pH}$ $4 \pm 0.05$ with methyl alcohol(50:50) operating at a flow rate of $1 \mathrm{ml} / \mathrm{min}^{36}$.

\section{Statistical treatment of data}

The average plasma Cefixime concentration profiles were produced. Statistical comparisons were performed using Graph pad prism for Windows Version 7. For assessing two mean values for in vivo data for statistical significance (at $\alpha=0.05$ level of significance) was done by utilizing 2-tailed unpaired test for samples with unequal variance where HPBCD-IC was analyzed with the Cefixime (CFX).

\section{Results and discussion}

\section{Phase solubility study}

The phase solubility diagram for drug-HPBCD is given in figure 1. It was seen that the solubility of drug increases with increase in complexing agent i.e. HPBCD in linear fashion. According to Higucchi and Connar, as the slope is less than one for the phase solubility profile, thus it is $A_{L}$ type. The slope was less than one i.e. 0.08483 lead to conclusion of formation of 1:1 stoichiometry for complextion of drug with HPBCD (33). The apparent stability constant for the drug-HPBCD complexation was found to be 73.94 per Mole. The stability constant's (Ks) value ranging from 50 to $5000 \mathrm{M}^{-1}$ are regarded as appropriate for the enhancement of solubility and stability of poorly water soluble drugs ${ }^{37}$.

\section{Solubility studies}

Saturation solubility study was conducted to evaluate the effect of polymer on aqueous solubility of Cefixime. All the test samples showed an increase in drug solubility over crystalline CFX. The solubility profile of Cefixime, physical mixture and Inclusion complexation of Cefixime with polymers are shown in figure 2. Plain Cefixime was slightly soluble in water. The solubility of plain Cefixime and its physical mixture in water was less as compared to Inclusion complexations prepared by other method.

It was inferred that aqueous solvency of all the complexes was enhanced when the CFX was complexed with $\mathrm{HP} \beta-\mathrm{CD}$ in 1:1 molar proportion. The complex made in 1:1 molar proportion (independent of the technique used) demonstrated better solubility when contrasted with the complex made in 1:2 molar proportion. Thus this ratio is optimum as further increase did not show solubility enhancement for Cefixime irrespective of the technique used for complexation.

The solubility of inclusion complex with HP $\beta$-CD prepared by spray drying method in distilled water was found to show, an almost 8.2 fold increase in solubility as compared to pure drug. The solubility of binary systems (Complexing agent and drug) was found to be increased in distilled water. Binary complexes with HP $\beta$ - 
CD exhibited greater increase in saturation solubility. The binary complex with HP $\beta$-CD prepared by PM, KD, SE, LYO, MW method showed a 2.71, 3.27, 4.13, 5.7, 4.54 fold increase respectively in distilled water.

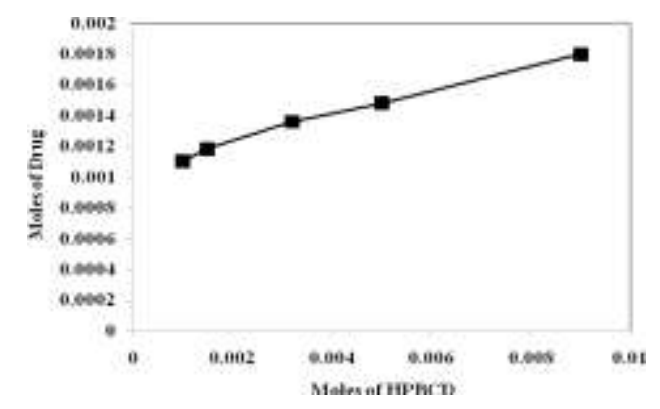

Figure 1: Phase solubility study of Cefixime with HPBCD

It was apparent that HP $\beta-C D$ had a marked effect in the solubility of Cefixime. Thus, spray drying method exhibited the highest solubility of Cefixime with $\mathrm{HP} \beta C D$, after that lyophilization, microwave irradiation, Solvent evaporation, kneading \& physical mixture $(\mathrm{p}<0.05)$.

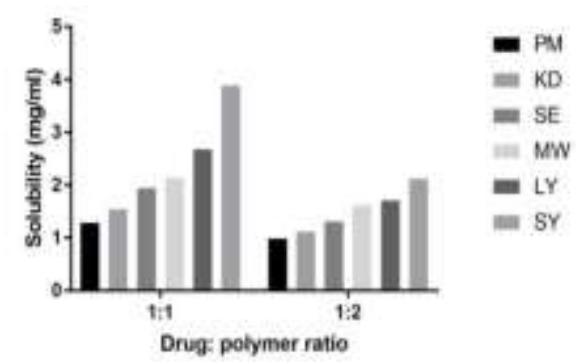

Figure 2: Saturated solubility using different solubilizing techniques with different drug: polymer ratio

\section{SEM}

The morphology of the CFX and IC was examined using SEM and the photographs are shown inFigure 3.SEM of pure CFX appeared as flat-broken needle-shaped crystals of varying sizes with well-developededges. Whereas its surface was modified after inclusion complexation preparation with carrier HPBCD \& by spray drying method ${ }^{38}$.

However, CFX- HP $\beta C D$ inclusion complex was appeared as spherical shaped particles of varying sizes with smooth surface though the topograpy of the original drug crystals was unlike than this prepared spray dried IC.

DSC

The thermal behavior of pure CFX and Inclusion complexation are shown in Figure 4. The DSC thermogram of pure CFX showed a melting endotherm at $215.64^{\circ} \mathrm{C}$ and for loss of water of hydration at $122.94^{\circ} \mathrm{C}$. For physical mixing, the DSC thermogram of IC is characterized by addition of individual components i.e. broad peak at $107.10^{\circ} \mathrm{C}$ and melting endotherm at $215.60^{\circ} \mathrm{C}^{39}$.

For kneading, the thermal curve showed peak at $87.74^{\circ} \mathrm{C}$ and melting endotherm was shifted to 195.67 ${ }^{0} \mathrm{C}$ while, for solvent evaporation, it showed dehydration peak at $88.49^{\circ} \mathrm{C}$ and melting endotherm was shifted to $204.33^{\circ} \mathrm{C}$. For lyophilization \& microwave irradiation it was found to be shifted to $184.75{ }^{\circ} \mathrm{C} \mathrm{\&} 190.02{ }^{\circ} \mathrm{C}$ respectively. However, DSC thermograms of HP $\beta$ CD spray dried inclusion complex showed broad \& negligible endothermic peak at $204.5^{\circ} \mathrm{C}$. The shifting as well as decrease in melting endotherm of the Cefixime in the 
spray-dried inclusion complex suggesting that the Cefixime is entrapped in amorphous or non-crystalline form in the cavity of the HPBCD. As shifting of melting endotherm has occurred, it can be said that stable inclusion of drug into cyclodextrin cavity has been formed. Another intriguing component of the thermograms is the wide endotherm going from room temperature to $110^{\circ} \mathrm{C}$, with a most extreme in the range of $85-95^{\circ} \mathrm{C}$ that likely relates to the loss of unequivocally bound water from the cyclodextrin hydration shell ${ }^{39}$. Inclusion complex formation utilizing spray drying displayed a largest decrease in enthalpy that is contributory to the increase in solubility determined in distilled water.

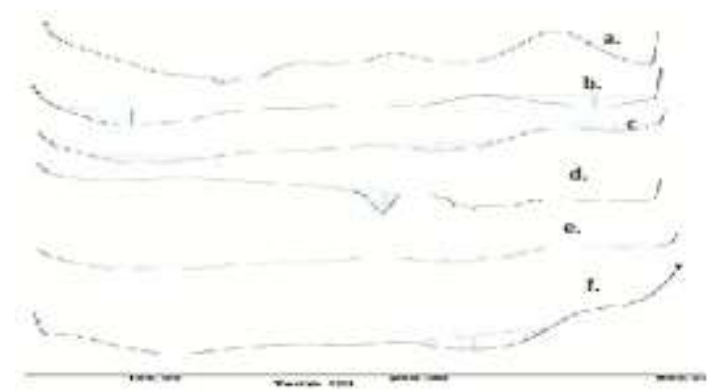

Figure 4: DSC thermograms of a. PM, b. KD, c. SE, d. LY, e. MW, f. SY

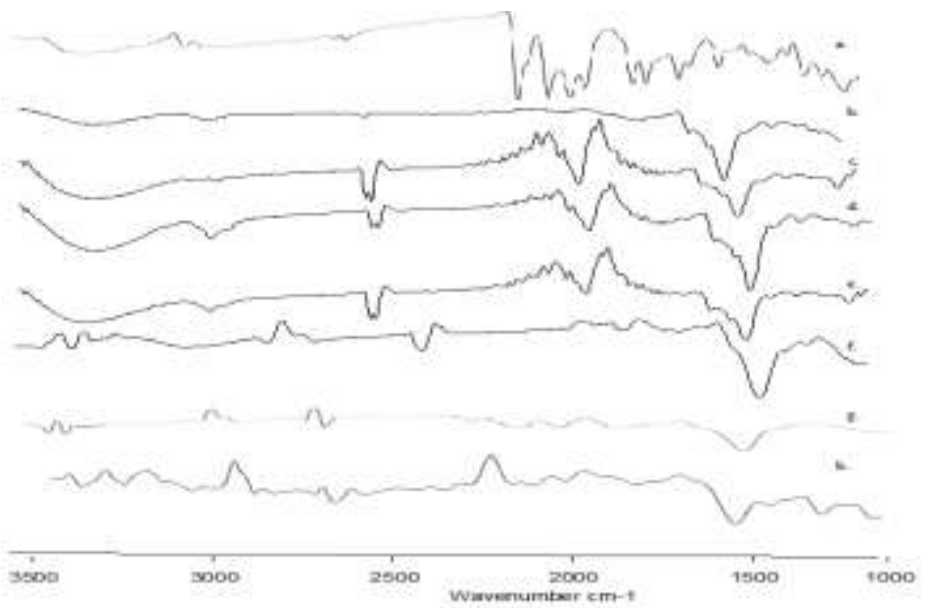

Figure 5: ATR spectra of a. Pure CFX, b. MW,c. LY, d. HPBCD Pure, e. KD, f. SE, g. PM, h. SY

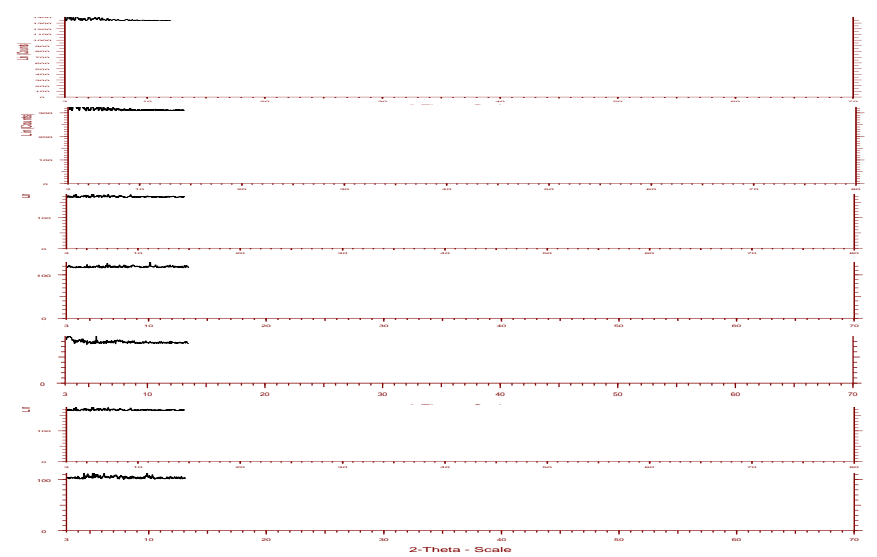

Figure 6: XRD spectras of a.Pure Cefixime, b. Physical mixture (PM), c. Solvent evaporation (SE), d. Kneading method (KD), e. Microwave irradiation (MW), f. Lyophilization (LY), g. Spray drying method (SY). 


\section{X-ray Diffraction (XRD)}

The X-ray diffraction images of CFX and IC are depicted in Figure 6. Pure CFX demonstrated typical diffraction peaks at $2 \theta$ positions of $5.89,8.97,19.55,24.745,26.36,27.34,31.88$ and 35.112. Pure CFX exhibited sharp and intense peaks in the range of $5^{\circ}-60^{\circ}$ at $2 \theta$ angles, which suggested that CFX was present in crystalline form. For physical mixing all the drug peaks were present only with slightly reduction of peak intensities thus indicating crystalline nature which may be the reason behind why physical mixing lead to little increment in solubility and dissolution rate of Cefixime.

But inclusion complexation prepared by solvent evaporation, microwave irradiation, lyophilization, kneading gave diffused XRD pattern with highly reduced peak intensities suggesting amorphous nature of IC. However, for the IC, the discriminatory peaks of CFX were clearly absent and halo pattern was observed for spray dried formulation indicating the absence of crystallinity. However, the XRD pattern of the spray drying complex with HPBCD was found to be diffused with peak intensities diminished greatly which in turn indicate reduction of crystallinity than that of pure cefixime confirming formation of new solid phase. The XRD pattern of spray drying complex is totally diffused, indicating the formed complex has an amorphous nature thus which may be reason for improvement of solubility and dissolution rate of poorly soluble Cefixime in inclusion complexation. Thus, spray drying technique found to prove best among all other solubility enhancing techniques for inclusion complex formation of Cefixime.

Release of enthalpy bearer-water molecules (their always ready to form $\mathrm{H}$ - bonding potential giving higher energy to the system) from the cavity of HPBCD is forcing to fit Cefixime in it and thus forming inclusion complexation ${ }^{40}$.

\section{ATR}

ATR spectroscopy was carried out for characterizing the interaction between Cefixime \& HPBCD in solid state. The ATR spectra revealed that there is no interaction between drug and carrier. The functional groups are not altered during mixing with carriers. The ATR spectra of Cefixime, Physical mixture and inclusion complexation were shown in figure 5. Pure Cefixime showed vibrational peaks of - $\mathrm{NH} 2$ primary amine at wave number $3288.56 \mathrm{~cm}^{-1}$, O-H stretching at $3526.59 \mathrm{~cm}^{-1}, \mathrm{C}-\mathrm{H}$ stretching at $2889.48 \mathrm{~cm}^{-1}, \mathrm{C}=\mathrm{O}$ stretch of CONH at $1664.84 \mathrm{~cm}^{-1}, \mathrm{~N}-\mathrm{H}$ bending at $1540.84 \mathrm{~cm}^{-1}, \mathrm{C}-\mathrm{N}$ stretching/ring stretching at $1590.81 \mathrm{~cm}^{-}$ ${ }^{1}$, C-N aromatic stretching at $1380.55 \mathrm{~cm}^{-1}$, Carbonyl stretching of $\mathrm{COOH}$ at $1766.16 \mathrm{~cm}^{-1}$, C-S stretching at $666.05 \mathrm{~cm}^{-1}$. For pure HPBCD ATR spectra, vibrational peaks were observed at $3398 \mathrm{~cm}^{-1}(\mathrm{O}-\mathrm{H}), 2925 \mathrm{~cm}^{-1}$ (C$\mathrm{H}), 1596.95 \mathrm{~cm}^{-1}$ (H-O-H bending), $1031.85 \mathrm{~cm}^{-1}$ (C-O-C, stretching). For physical mixing of Cefixime and HPBCD, ATR spectra showed peaks of both drug and HPBCD but were having reduced intensity. For the kneaded product also drug peaks were completely disappeared with reduced peak intensity suggesting that inclusion complex has been formed in solid state.

For HPBCD-IC prepared by solvent evaporation method, $\mathrm{OH}$ stretching at $3332.76 \mathrm{~cm}^{-1}$ while $\mathrm{C}=\mathrm{O}$ stretching at $1606.09 \mathrm{~cm}^{-1}$ were observed. The absorption band of carbonyl stretching in Cefixime was shifted to $1606.09 \mathrm{~cm}^{-1}$ because of intermolecular hydrogen bond formation between HPBCD and drug ${ }^{40}$. And, all the drug peaks were seem to be disappeared and lead to the conclusion that cephem ring of Cefixime which is having $\mathrm{COOH}$ as functional group attached to it might have trapped in the hydrophobic cavity of HPBCD and confirming inclusion complex formation. Inclusion complexes prepared by lyophilization, microwave irradiation and spray drying, showed that Cefixime prominent peaks were either disappeared or shifted, smoothen with reduced peak intensity but no new peak was observed suggesting that inclusion complexation has occurred but without any bond formation (i.e. Co-valent bond has not formed). The stretching vibration of above all mentioned functional group were found to be within range in all inclusion complexation as well as in physical mixture indicating absence of any significant chemical interaction in solid state. Thus, disappearance and shifting with smoothness of some peaks were noted in IR spectra of Inclusion complexation with HPBCD. It showed peaks of reduced intensity. Some peaks of Cefixime were disappeared indicating formation of complex in solid state. 


\section{In vitro drug release}

In vitro drug release profile of the different ICs of CFX in PBS pH 7.2 and the pure drug are compared in Figure 7. As can be seen, all the ICs were able to increase the dissolution rate of CFX. These data are in agreement with the increase in drug solubility in these formulations. There was significant difference for IC prepared by spray drying $(\mathrm{p}<0.001)$ when compared to other methods for the \%drug released at $5 \mathrm{~min}$.

However, \% drug release at 45 min, there was having no significant difference among lyophilization, microwave irradiation and spray drying method. It might be ascribed to the faster drug discharge in a short period. But overall, \% drug release was higher for spray drying method which leads to its selection for further study. Dissolution rate enhancement for spray drying may be due to diminished crystallinity as indicated by its XRD pattern. DSC results also showed that the amorphous nature of IC leads to the improvement of in vitro dissolution characteristics of Cefixime in solid complex.

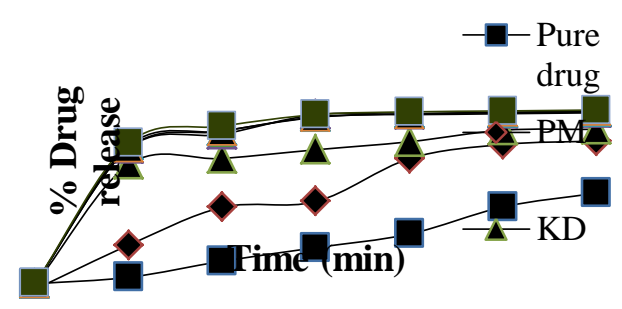

Figure 7: Dissolution profile of different solubilizing techniques used for complexation

HPBCD also acts as hydrophile with somewhat surfactant like properties which may be contributing for dissolution rate enhancement in binary systems. Increased hydrophilicity of resultant product may have lead to increased dissolution with increased wetting by HPBCD of Cefixime ${ }^{41}$. HPBCD's ability in forming stable inclusion complex may have added to the dissolution enhancement of Cefixime. IC prepared by spray drying method increased dissolution rate because in spray drying drug particles are broken down into smaller particles thus increasing its total surface area coming into contact with dissolution media. For kneaded products, trituration along with little solvent makes smaller particles with total increased surface area for dissolution. Products made by solvent evaporation, are made by mechanical stirring of drug and polymer along with its dissolution in respective solvents thus its forming smallest drug particles with increased total surface area. In microwave irradiation, drug particles and polymer are dissolvent in respective solvents along with proper collisions between them by microwave and thus forming smaller drug particles with well formed drug-polymer complex. Freeze drying involves dissolution of drug and polymer in respective solvents, freezing and then freezing drying for removal of solvents giving amorphous powder with smaller drug particles and ultimately improved dissolution.

Thus, it can be said that as IC prepared by spray drying method were able to release drug early than that of other methods leading to increased antibacterial activity of Cefixime as can be seen by antibacterial studies. The low apparent stability constant as calculated by phase solubility studies show that IC prepared with HPBCD is having high energy i.e. it is less stable and hence releases drug easily when it comes in contact with dissolution medium and thus increasing its antibacterial activity. Pure CFX exhibited a low dissolution rate, with a $43.52 \%$ release, in $30 \mathrm{~min}$ and only reached $51.37 \%$ after $45 \mathrm{~min}$.

Physical mixture did not show any significant improvement in dissolution rate. This result attributed to hydrophobic nature of drug due to which drug particle floats on the surface of the dissolution medium and prevents its intimate contact with the dissolving medium. In-vitro dissolution studies revealed that the inclusion complexes by spray drying method released the drug to a greater extent as compared to CFX. Binary complex with $\mathrm{HP} \beta C D$ showed a release of $97.33 \%$ in $\mathrm{pH} 7.2$ buffer in $20 \mathrm{~min}$. 
Drug release was higher in case of binary complex with HP $\beta C D$ by spray drying i.e. $98.40 \%$ in $\mathrm{pH} 7.2$ buffer in 45 min. The formulations with HPBCD prepared by PM, KD SE, LY methods exhibited a release of $70.9 \%, 80.44 \%, 95.77 \%$, and $96.69 \%$ respectively in $20 \mathrm{~min}$. The formulations with MW $(96.24 \%)$ in $20 \mathrm{~min}$ and SY showed similar release curves and higher dissolution rates than the pure CFX and other formulations. Therefore, the formulation SY with $98.40 \%$ release in 45 min was chosen for further study.

For the binary systems, dissolution rate improvement may be due to complex formation, increased wetting, increased mechanical contact between drug and complexing agent (42). HPBCD-IC prepared by spray drying method showed complete drug release and high solubility compared to earlier method hence it was further characterized.

\section{H NMR \& 2D (NMR) ROSEY studies}

Keeping in mind the end goal to investigate the conceivable incorporation mechanism of the CFX/HP $\beta C D$ complex, we analyzed the 1H NMR spectra of CFX - HPBCD IC with pure CFX and HPBCD (Figure 8). Attributable to CFX poor water dissolvability, D2O was utilized as a dissolvable solvent for $1 \mathrm{H}$ NMR. Thus, the inclusion complex was characterized by 1D 1H NMR and 2D ROESY NMR analysis. 1H NMR was used to confirm formation of inclusion complex in solution. The drug compound as guest in cyclodextrin as host more often has protons upfield and guest protons as downfield. Thus, we can elucidate association and inclusion of drug in cyclodextrin ${ }^{44,45}$.

The prima CFX protons display chemical displacement at $\delta 7.5-5.5 \mathrm{ppm}$, differing from the HP $\beta C D$ 's protons $(\delta 1.0-5.5 \mathrm{ppm})$. The interaction regions of these protons used to compare with that of the HP $\beta C D$ 's H1 protons, which leads to ascertion of the incorporation stoichiometry of the CFX/HP $\beta C D$ complex, \& is 1:1.

Table 1: The chemical shifts ( $\delta$ ) of HPßCD and CFX/HPßCD complex

\begin{tabular}{|l|l|l|}
\hline & HP- $\beta-C D$ & CFX/HP- $\beta-C D$ complex \\
\hline H1 & 4.99 & 4.92 \\
\hline H2 & 3.54 & 3.49 \\
\hline H3 & 3.87 & 3.872 \\
\hline H4 & 3.49 & 3.48 \\
\hline H5 & 3.77 & 3.805 \\
\hline H6 & 3.77 & 3.864 \\
\hline
\end{tabular}

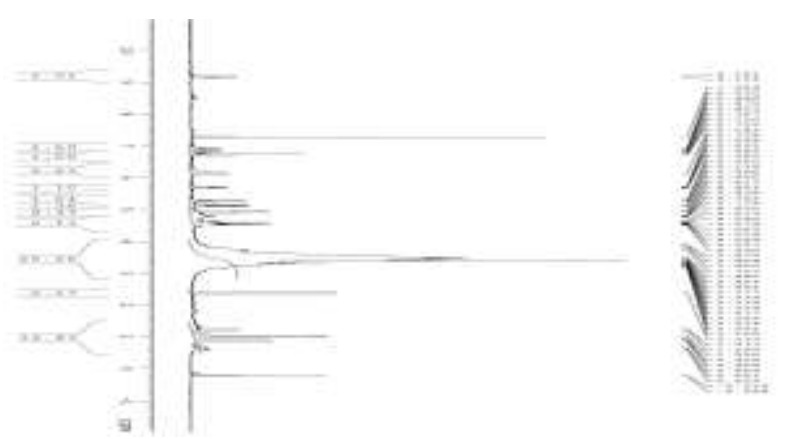

Figure 8: 1H NMR spectra of Solid complex 


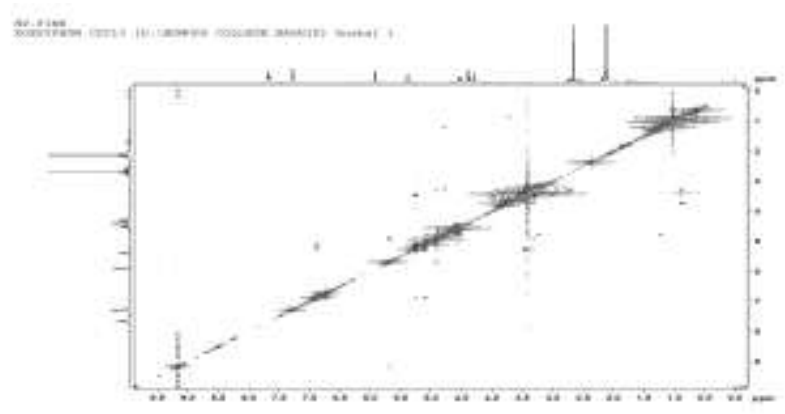

\section{Figure 9: The 2D 1H NMR (ROESY) spectra of inclusion complex}

NMR study, where protons are molecularly interacted, leading to change in NMR frequencies of CFX and HP- $\beta-C D$ in 1 H-NMR spectrum (Figure 8). Chemical shift change were displayed in the NMR of HP- $\beta$ $\mathrm{CD}$ region $(\delta 3.5 \tilde{n} 3.8)$, giving information on mode of inclusion ${ }^{46}$. The H-1 and H-4 protons of HP- $\beta-C D$ show downfield movement, may be due to some conformational alteration of essential - OH assemble, \& modification in polarity stimulated by the incorporation of $\mathrm{CFX}^{47}$. The H-3 and H-5 protons of HP- $\beta-\mathrm{CD}$ showing upfield move in the spray dried IC, suggesting their presence near to the $\pi$-electron cloud of a aromatic core $\&$ its magnetic anisotropy. These outcomes showed that CFX was entrapped in the hole of HP- $\beta$-CD (47). Inclusion complex formation with HPBCD leads to shift of the NMR signals of HPBCD towards upfield \& drugs NMR signals shifting downfield indicating inclusion complex formation. HPBCD's protons located inside the cavity like H3, H5/H6 \& where, H5/6 NMR signals show more sifts upfield; stating that Cefixime's entrapment in HPBCD's cavity by narrow rim \& inclusion complex formation. Two-dimensional (2D) NMR spectroscopy provide important data of the spatial nearness between host and guest through intermolecular dipolar cross-connections ${ }^{47}$. Two protons close situated in space can create nuclear overhauser impact (NOE) cross-correlation in NOE spectroscopy (NOESY) or ROESY. The existence of NOE cross-crests between protons from two species shows spatial contacts inside $0.4 \mathrm{~nm}$ (48). To acquire conformational data, we got 2D ROESY of the CFX- HP $\beta C D$ inclusion complex. 2D-ROSEY was used to see spatial proximity between atoms. It was used to see cross peaks between Cefixime and HPBCD (Figure 9). 1H NMR and ROESY spectra results showed a cross-peak between H-5 proton of HP $\beta C D$ and the aromatic rings group of Cefixime. This revealed that the hydrophobic interactions between aromatic rings of Cefixime and the cavity of HP $\beta C D$.

Evaluation of the CFX-HPBCD complex by 1H NMR obviously showed the nearness of the structure protons of the CFX atom, thus supports the enhanced solubility of CFX by HPBCD complexation.

In view of these conclusions, together with the 1:1 stoichiometry concluded by NMR spectra we proposed the conceivable inclusion modes for the CFX/HP $\beta C D$ as outlined in Figure 10. The intermolecular insertion of Cefixime into hydrophobic cavity of HP- $\beta-C D$ resulted in change of NMR shifts while cross peaks supports proposed mode of inclusion of Cefixime into HPBCD host cavity.

\section{Molecular Docking Simulations}

A docking count can be utilized as the hypothetical base to anticipate incorporation mode $(49,50)$. So as to justify the NMR exploratory outcomes, CFX was docked into HP $\beta C D$ utilizing Schrodinger programming ( Inc. USA). The work uncovered a minimum energy favored ultimate insertion for the complex. The best preferred conformer got for HP $\beta C D$ and CFX in docking study is appeared in Figure 11. The consequences of the docking studies are given as G-score, Energy, Good, Bad and also Ugly Van Der Waals (vdw) interactions. The G-scores are given as negative qualities, proposing that progressively the negative values more are the binding interactions ${ }^{34}$. The docking studies were done for the designed Cefixime and HPBCD protein. The best conformer showed G-score of -6.024. The designed complexes were found to show great restricting partiality to the receptor. Yellow specked line point H-bond connections with receptor. Conceivable H-holding connections are appeared by green specked line. The outcomes are in great agreement with that got by the NMR analyze. The presence of a hydrogen bond between the $\mathrm{COO}-\mathrm{H}$ hydrogen of Cefixime and neighboring $\mathrm{O}-\mathrm{H}$ oxygen of 
HPBCD (spoke to by specked bolt) demonstrate the estimation of hydrogen bonding in the stability of the inclusion complex. The 3D representation forecast of the incorporation demonstrating the addition of aromatic part of CFX in HP- $\beta-C D$ ) and its minimum energy conformers has been represented (Figure 12). Molecular docking studies demonstrated that Cefixime can make stable inclusion complexation getting different conformers.

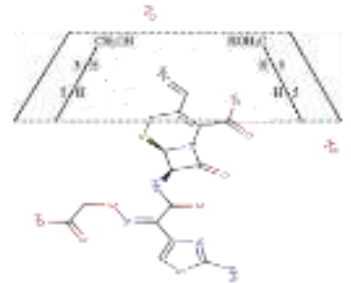

Figure10. Representation of the $\mathrm{CFX} / \mathrm{HPbCD}$ inclusion complex,showing the CFX structure partially inserted in the HPbCD cavity.
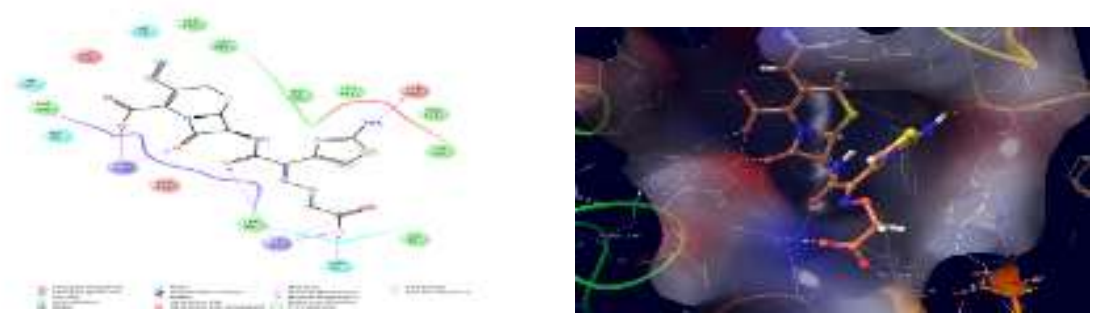

Figure 11\&12: 2D\& 3D Representation of mode of inclusion of Cefixime inside HPBCD cavity

\section{Antimicrobial studies}

The antimicrobial activity of binary systems of CFX with HPBCD against Gram-positive (S. aureus) and Gram-negative (E. coli) species was checked by disk diffusion method and compared with the pure CFX. The zone of inhibition can be seen in figure 13. These studies revealed that binary systems of CFX have shown greater antimicrobial activity than CFX alone. However, CFX-HPBCD spray dried has shown significant and highest zone of inhibition against both the microorganisms as compared to pure CFX alone and other binary systems. The greater antimicrobial activity of CFX-HPBCD spray dried may be due to the ability of HPBCD to release the drug readily from the inclusion complexation ${ }^{43}$.
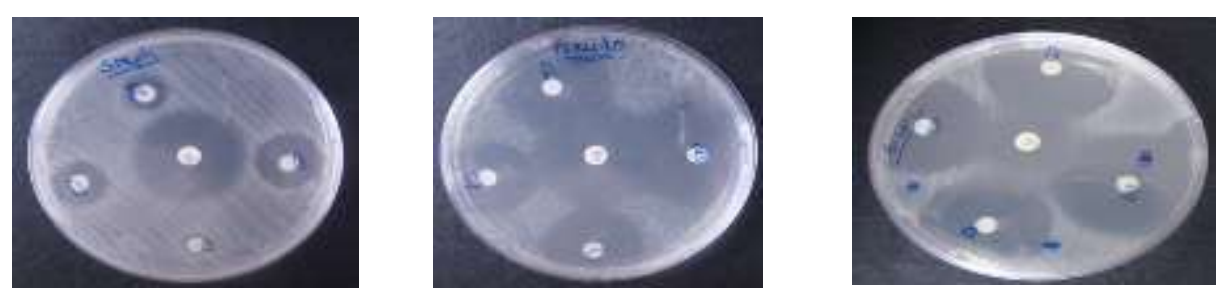

Figure 13: Antimicrobial study: zone of inhibition. Staphylococcus aureus a.Pseudomonas aerogenosa, $b$. E. coli. Where A: HPBCD LY, B: HPBCD SY, D: HPBCD MW.

\section{In-Vivo absorption study in wistar rats:}

The mean plasma concentration time profile for CFX following oral administration of pure CFX and CFX-IC are shown in Figure 14. and the pharmacokinetic parameters are summarized in Table 2. The differences in the shape of the concentration time profile between the two treatments were found, expressed by higher Cmax and tmax for the HPBCD-IC. Dosing the aqueous suspensions of Cefixime resulted in the average 
Cefixime plasma concentrations. However, the AUC was 3 times greater when Cefixime was administered as IC, compared with the AUC obtained for the aqueous Cefixime suspension. The $\mathrm{T}_{\max }(4 \mathrm{hr})$ after IC dosing was different than the $\mathrm{T}_{\max }$ obtained within aqueous suspensions $(3 \mathrm{hr})$. The differences between the two treatments for Cmax $(p<0.0001)$ and tmax $(p=0.03)$ were statistically significant. These results reveal that formulation of Cefixime as IC results in a significantly $(\mathrm{p}=0.05)$ increased absorption of Cefixime, compared with that from the aqueous suspensions. Based on these results, it can be concluded that the rapid and enhanced absorption of CFX obtained from the HPBCD-IC, with higher Cmax and tmax, could be due to improved dissolution resulting from reduced particle size, increased surface area, enhanced wettability, formation of high energy amorphous form \& inclusion complexation.

Table 2: Pharmacokinetic parameters following oral administration of inclusion complexation and CFX

\begin{tabular}{|l|l|l|}
\hline Parameter & CFX & IC \\
\hline $\begin{array}{l}\mathrm{AUC}_{0-12 \mathrm{~h}} \\
\mu \mathrm{g} \cdot \mathrm{h} / \mathrm{mL}\end{array}$ & 11.28 & 35.41 \\
\hline $\mathrm{C}_{\max } \mu \mathrm{g} / \mathrm{mL}$ & $1.365 \pm 0.01607$ & $4.87 \pm 0.471$ \\
\hline $\mathrm{T}_{\max } \mathrm{h}$ & 3 & 4 \\
\hline
\end{tabular}

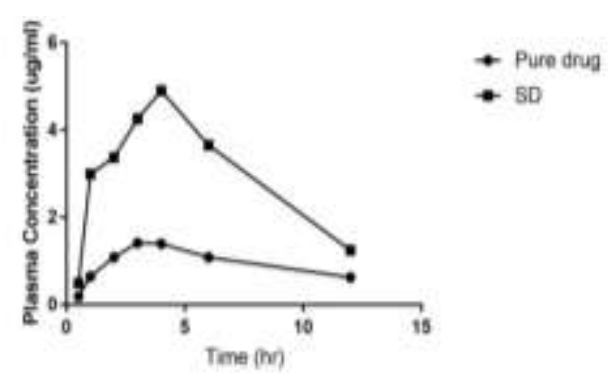

Figure 14: Plasma concentration-time profiles following oral administration of inclusion complexation and CFX $(n=6)$.

\section{Conclusions}

In conclusion, in this study we can say that inclusion complexation has formed in the solid state between Cefixime and HPBCD. Phase solubility studies aided in calculating apparent stability constant for HPBCD with Cefixime. The stoichiometry for the inclusion complexation was found to be 1:1. Different solubility enhancement methods like physical mixing; kneading, solvent evaporation, microwave irradiation, lyophilization, and spray drying can be used for inclusion complex formation. Different methods thus due to difference in procedures thus gave different effect on solubility and rate of dissolution of poorly soluble Cefixime. Thus, spray drying method showed significant difference $(\mathrm{p}=0.05)$ in solubility as well as rate of dissolution as compared to pure Cefixime. The increase in dissolution may be due to increase in total surface area due to smaller particles generated during spray drying, increased mechanical contact between drug and HPBCD, with increased wetting and hydrophilicity. The change of crystalline Cefixime to amorphous form as indicated by DSC, XRD studies. Inclusion complex formation in solid state confirmed by ATR, NMR studies and later 3D ROSEY study gave interesting insights into mechanism of inclusion. Molecular docking studies gave idea about 3D and 2D conformation with stable conformer and stoichiometry between Cefixime and HPBCD. Pharmacokinetic studies and antibacterial studies confirmed that spray drying method is best suited method for inclusion complex formation for poorly soluble Cefixime and increasing its solubility and ultimately oral bioavailability.

On the basis of the in-vitro dissolution and in-vivo pharmacokinetic studies, it was concluded that a formulation of Cefixime with HPBCD by spray drying method has been developed with enhanced bioavailability \& reduction of dose with increasing patient compliance. This study emphasizes the use of 
comparative study of different solubility enhancement techniques for the best formulation development of poorly soluble drug like Cefixime. Hence, it can be concluded that selection of polymer and method of solubility enhancement are critical for the formulation development which consequently affects oral bioavailability of drug. For the development of formulation for poorly soluble drug, depending on experimental data, it is concluded that the type of solubility enhancement techniques and type of drug-complexing agent ratio are the critical factors.

\section{Acknowledgments}

The authors would like to thank the Sophisticated Analytical Instrumentation Facility (SAIF) at Cochin University, Kochi for the XRD, SEM studies\& MGV College, Nashik for doing DSC Study. We would also like to thank Savitribai Phule Pune University, Pune for doing 1H NMR \& 2D ROSEY analysis. I am also thankful to the Glenmark pharmaceuticals, Sinnar, Nashik for providing gift samples required for the research work.

\section{References}

1. $\quad$ S. Jambhekar and P. Breen, Drug Discov. Today, 21, 363 (2016); doi:10.1016/j.drudis.2015.11.016.

2. M. di Cagno, Molecules, 22, 1 (2017); doi:10.3390/molecules22010001.

3. $\quad$ L. Leclercq, Beilstein J. Org. Chem., 12, 2644 (2016); doi:10.3762/bjoc.12.261.

4. https://clinicaltrials.gov/ct2/results?term=cefixime\&pg=1.

5. https://clinicaltrials.gov/ct2/show/NCT01949363?term=cefixime\&rank=1.

6. $\quad$ "WHO Model List of Essential Medicines (19th List)" (PDF). World Health Organization. April 2015. Retrieved 8 December 2016.

7. $\quad$ SUPRAX Package Insert" (PDF). Lupin Pharmaceuticals, Inc. March 2013. Retrieved 7 April 2016.

8. Y. Matsumoto, A. Ikemoto, Y. Wakai, F. Ikeda, S. Tawara and K. Matsumoto, Antimicrob. Agents Chemother., 45, 2450 (2001); doi:10.1128/AAC.45.9.2450-2454.2001.

9. Y. Matsumoto, A. Ikemoto and S. Tawara, J. Infect. Chemother., 5, 176 (1999); doi:10.1007/s101560050030.

10. S.C. Sweetman, (1999). In Martindale, The Complete Drug Reference. London: Pharmaceutical Press, London, 32nd ed., 165-166.

11. USPDI, Drug information for the health care professional. The United States Pharmacopeial Convention, Inc. 15th edition, Taunton, MS 1995, 671-678.

12. L. Harcouet, D. Lebrec, C. Roze, C. Carbon and R. Farinotti, J. Pharmacol. Exp. Ther., 281, 738 (1996).

13. R.D. Faulkner, P. Fernandez, G. Lawrence, L.L. Sia, A.J. Falkowski, A.I. Weiss, A. Yacobi and B.M. Silber, J. Clin. Pharmacol., 28, 700 (1988); doi:10.1002/j.1552-4604.1988.tb03203.x.

14. A. Tsuji, H. Hirooka, T. Terasaki, I. Tamai and E. Nakashima, J. Pharm. Pharmacol., 39, 272 (1987a); doi:10.1111/j.2042-7158.1987.tb06265.x.

15. A. Tsuji, T. Terasaki, I. Tamai and H.H. Hirooka, J. Pharmacol. Exp. Ther., 241, 594 (1987B).

16. A.H. Dantzig, L.B. Tabas, L. Bergin, BiochimicaetBiophysicaActa, 1112, 167(1992).

17. A.H. Dantzig, D.C. Duckworth and L.B. Tabas, BiochimicaetBiophysicaActa, 1191, 7 (1994).

18. T. Kimura, T. Yamamoto, M. Mizuno, Y. Suga, S. Kitade and H. Sezaki, J. Pharmacobiodyn., 6, 246 (1983); doi:10.1248/bpb1978.6.246.

19. K.I. Inui, M. Yamamoto and H. Saito, J. Pharmacol. Exp. Ther., 261, 195 (1992). 
20. B. Brahmaiah, K. Sreenivasulu and M. Rajwardanreddy, Int. J. Pharm. Therap., 4, 140 (2013).

21. S. Arora, P. Sharma, R. Irchhaiya, A. Khatkar, N. Singh and J. Gagoria, Int. J. Drug Develop. Res., 2, 424 (2010).

22. S. Aleti, D. Rangaraju, A. Kant, M. Shankraiah and J. Venkatesh, Int. J. Res. Pharm. Chem., 1, 283 (2011).

23. D. Saindane, A. Kulkarni, A. Sagri, T. Khade and M. Patil, Int. J. Pharm. Technol., 1, 149 (2010).

24. U. Varia, C. Patil, N. Kalyane and P. Agrawal, J. Pharm. Res., 3, 570 (2010).

25. S. Mallick, A. Mondal and S. Sannigrahi, J. Pharm. Pharmacol., 60, 833 (2008); doi:10.1211/jpp.60.7.0004.

26. S. Verma, A. Kumar, V. Malik and V. Kumar, Der. Pharma. Sinica, 4, 8 (2013).

27. S. Seetharaman, Int. J. Pharmaceut. Clinical Res., 8, 179 (2016).

28. M. Haritha and B. Pragatikumar, J. Chem. Pharmaceut. Sci., 6, 234 (2013).

29. P. Mehta, A. More and A. Kulkarni, Int. J. Curr. Pharmaceut. Res., 5, 66 (2013).

30. P. Jadhav, B. Petkar, Y. Pore, A. Kulkarni and K. Burade, Carbohydr. Polym., 98, 1317 (2013); doi:10.1016/j.carbpol.2013.07.070.

31. T. Higuchi and K.A. Connors, Adv. Ana.l Chem. Instrum., 4, 117 (1965).

32. D.S. Saindane, Int. J. Pharm. Technol., 1, 149 (2010).

33. D. Bramhane, P. Kulkarni, E. Martis, R.R.S. Pissurlenkar, E.C. Coutinho and M.S. Nagarsenker, J. Pharm. Bioallied Sci., 8, 161 (2016); doi:10.4103/0975-7406.171680.

34. R. Bamane, T. Chitre and V. Rakholiya, Der. Pharma. Chemica, 3, 227 (2011).

35. S. Setthacheewakul, S. Mahattanadul, N. Phadoongsombut, W. Pichayakorn and R. Wiwattanapatapee, Eur. J. Pharm. Biopharm., 76, 475 (2010); doi:10.1016/j.ejpb.2010.07.011.

36. N. Jain, H. Jain and P. Jain, Technol. Arts Res. J., 3, 93 (2015); doi:10.4314/star.v3i4.14.

37. S. Khan, H. Batchelor, P. Hanson, Y. Perrie and A.R. Mohammed, J. Pharm. Sci., 100, 4281 (2011); doi:10.1002/jps.22613.

38. M. Shah, Y. Pore, S. Dhawale, K. Burade and B. Kuchekar, J. Incl. Phenom. Macrocycl. Chem., 76, 391 (2013); doi:10.1007/s10847-012-0211-z.

39. T. Pralhad and K. Rajendrakumar, J. Pharm. Biomed. Anal., 34, 333 (2004); doi:10.1016/S07317085(03)00529-6.

40. H. Hatanaka, F. Komada, Y. Mishima and K. Okumura, J. Pharmceut. Sci., 82, 1054 (1993); doi:10.1002/jps.2600821014.

41. L. Shan-Yang and K. Yuh-Horng, Int. J. Pharm., 56, 249 (1989); doi:10.1016/0378-5173(89)90022-7.

42. M.V.G. de Araújo, E.K.B. Vieira, G.S. Lázaro, L. de Souza Conegero, O.P. Ferreira, L'.E. Almeida, L.S. Barreto, N.B. da Costa Jr. and I.F. Gimenez, Bioorg. Med. Chem., 15, 5752 (2007); doi:10.1016/j.bmc.2007.06.013.

43. O. Aleem, B. Kuchekar, Y. Pore and S. Late, J. Pharm. Biomed. Anal., 47, 535 (2008); doi:10.1016/j.jpba.2008.02.006.

44. P. Demarco and A. Thakkar, J. Chem. Soc., 11, 2 (1970).

45. B. Pose-Vilarnovo, I. Perdomo-López, M. Echezarreta-López, P. Schroth-Pardo, E. Estrada and J. Torres-Labandeira, Eur. J. Pharm. Sci., 13, 325 (2001); doi:10.1016/S0928-0987(01)00131-2.

46. V. Sinha, A. Nanda, R. Chadha and H. Goel, Drug Res., 68, 585 (2011). 
47. C.M. Fernandes, R.A. Carvalho, S. Pereira da Costa and F.J.B. Veiga, Eur. J. Pharm. Sci., 18, 285 (2003); doi:10.1016/S0928-0987(03)00025-3.

48. D. Neuhaus and M. Williamson, The nuclear Overhauser effect in structural and conformational analysis, 2nd ed.; VCH-Publishers: New York, 1989.

49. J.M. Choi, E. Cho, B. Lee, D. Jeong, Y. Choi, J.-H. Yu and S. Jung, Carbohydr. Polym., 151, 40 (2016); doi:10.1016/j.carbpol.2016.05.046.

50. L. Yang, S. Wang, S. Zhou, F. Zhao, Q. Chang, M. Li, W. Chen and X. Yang, Mater. Sci. Eng. C, (2017). 